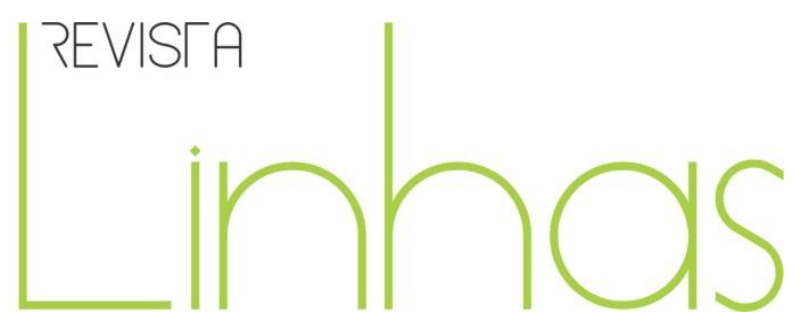

\title{
Algunas reflexiones acerca del "santuario" escolar, el conocimiento, las tecnologías digitales e Internet en el capitalismo informacional ${ }^{1}$
}

\begin{abstract}
Resumen
La construcción de la institución educativa como espacio de lo "sagrado", junto con la producción y reproducción de un tipo de conocimiento, y la incorporación de tecnologías digitales e Internet han motivado a grandes rasgos las líneas que siguen. Específicamente, en esta oportunidad, nos proponemos reflexionar acerca de la relación entre la institución educativa, más específicamente del programa institucional planteado en términos de Dubet, Francois (2006), y la producción de conocimiento con ocasión de las tecnologías digitales e Internet. En otras palabras, deconstruir y reconstruir la problemática de la producción y reproducción de conocimiento en la institución educativa contemporánea con ocasión de tecnologías digitales e Internet, comprender algunas de las transformaciones actuales en contextos más amplios y describir desafíos presentes y futuros, es nuestro propósito.
\end{abstract}

Palabras clave: Programa institucional; Tecnologías digitales e Internet; Capitalismo informacional y conocimiento.

\author{
Lucila Dughera \\ Doutora em Ciências Sociais pela \\ Faculdade Latino-Americana de \\ Ciências Sociais - FLACSO - \\ Argentina \\ ludughera@gmail.com
}

\section{Para citar este artigo: \\ DUGHERA, Lucila. Algunas reflexiones acerca del "santuario" escolar, el conocimiento, las tecnologías digitales e Internet en el capitalismo informacional. Revista Linhas. Florianópolis, v. 17, n. 33, p. 176-188, jan./abr. 2016.}

\section{DOI: $10.5965 / 1984723817332016176$}

http://dx.doi.org/10.5965/1984723817332016176

\footnotetext{
${ }^{1}$ Una versión preliminar de este escrito se presentó en el Seminario de Doctorado "¿A qué llamamos educar hoy? La época y los conceptos centrales de la pedagogía", dictado por el Prof. Estanislao Antelo en el marco del Doctorado en Cs. Sociales - Facultad Latinoamericana de Ciencias Sociales - FLACSO, sede Bs.As.
} 


\section{Some thoughts on the school "shrine", knowledge, digital technologies and Internet in information capitalism}

\begin{abstract}
The construction of the educational institution as space of the "sacred", along with the production and reproduction of a type of knowledge, and the incorporation of digital technologies and the Internet have motivated broadly speaking the lines that follow. Specifically, in this opportunity, we intend to reflect on the relationship between the educational institution, more specifically the institutional program raised in terms of Dubet, François (2006), and the production of knowledge at the time of the digital technologies and the Internet. In other words, deconstruct and rebuild some of the current transformations in broader contexts understand the problems of the production and reproduction of knowledge in the contemporary educational institution on the occasion of digital technologies and the Internet, and describe challenges present and future, it is our purpose.
\end{abstract}

Keywords: Institutional program; Digital technologies and Internet; Informational capitalism and knowledge.

\section{Algumas reflexões sobre o "santuário" escolar, o conhecimento, as tecnologias digitais e a Internet no capitalismo informacional}

\begin{abstract}
Resumo
A construção da instituição educativa como espaço do "sagrado", junto com a produção e reprodução de um tipo de conhecimento, e a incorporação de tecnologias digitais e a Internet motivaram de modo geral as linhas que seguem. Especificamente, nesta oportunidade, propor-nos-emos a refletir sobre a relação entre a instituição educativa, mais especificamente sobre o programa institucional, colocado nos termos de Dubet, François (2006), e a produção de conhecimento por ocasião das tecnologias digitais e a Internet. Em outras palavras, desconstruir e reconstruir a problemática da produção e reprodução de conhecimento na instituição educativa contemporânea por ocasião de tecnologias digitais e a Internet, compreender algumas das transformações atuais em contextos mais amplos e descrever desafios presentes e futuros, é nosso propósito.
\end{abstract}

Palavras-chave: Programa institucional; Tecnologias digitais e Internet; Capitalismo informacional e conhecimento. 
La problemática de la institución educativa en su conjunto, y su relación con la (re)producción y circulación de conocimiento ${ }^{2}$, no es una novedad del nuevo milenio. Sin embargo, al realizar un recorrido rápido por la bibliografía especializada, es posible observar que la crisis de los programas institucionales de Dubet, Francois (2006), o las transformaciones en puerta de las formas de lo escolar de Baquero, Ricardo; Diker, Gabriela y Frigerio, Graciela (2007), denotan y tienen anclaje en un tiempo específico que es entendido, desde nuestra perspectiva, como capitalismo informacional (Castells, Manuel 1997) o cognitivo (Bloundeau, Olivier 1999). Así, es posible señalar que las transformaciones acaecidas desde la década del '70 continúan situándose en el modo de producción capitalista y, junto con él, a todos y cada uno de los infortunios que ello implica. A la vez, que el conocimiento, al igual que en otras etapas históricas, sigue jugando un rol central. Claro que con el advenimiento de las tecnologías digitales ${ }^{3}$ e Internet se convierte en el principal insumo y motor de los procesos productivos (Castells, 1997).

Esta sucinta contextualización no intenta, ni mucho menos pretende, ser exhaustiva, sino simplemente ansía de mínima recuperar algunas de las características del devenir social actual y, en cierto sentido, institucional.

Hechas estas salvedades, estamos en condiciones de discurrir acerca de la institución educativa en el siglo XXI. Problemática que nos coloca de cara frente a una cuestión que, por cierto, creemos se amalgama con ella y, a la vez, la excede, tal como lo es la producción y circulación de conocimiento. Sobre estos dos ejes descansan las próximas páginas, si bien cada uno podría ser, como lo ha sido, motivo de libros y debates, aún no clausurados, aquí nos interesa esbozar potenciales relaciones entre los mismos y las tecnologías digitales e Internet. Claro que si el lector está en busca de

\footnotetext{
${ }^{2}$ Existen diferentes concepciones acerca del conocimiento. Para profundizar en dicha temática, véase Zukerfeld, Mariano (2010).

${ }^{3}$ De la diversidad de tecnologías que se pueden encontrar dentro de la institución escolar nos interesa destacar las tecnologías digitales (ordenadores, páginas web, Internet), que "son aquellas que procesan, transmiten, almacenan o generan información digital (ID). La definimos como toda forma de conocimiento codificado binariamente mediante señales eléctricas de encendido-apagado" (Zukerfeld, Mariano 2007: 41).
} 
respuestas, lejos sea éste el "espacio" para lograrlo. Sí, en cambio, el lugar para intercambiar y extrapolar miradas respecto de estas cuestiones.

Para ello se divide al texto en cuatro apartados. En la primera sección se recupera la propuesta de Dubet, Francois (2006) acerca del programa institucional y se lo problematiza a la luz del capitalismo informacional. En el segundo apartado se figura el papel del conocimiento y la tan marcada distinción entre el saber escolar y saber "sabio". Ya en la tercera sección se intenta una posible relación entre los nodos de cada uno de los apartados anteriores y las tecnologías digitales Internet. Para concluir, en la cuarta sección, se establecen algunas posibles aberturas que, más que cerrar lo planteado, sugieren potenciales líneas de interrogación, que habiliten este continuar pensando.

En suma, deconstruir y reconstruir la problemática de la producción, reproducción y circulación de conocimiento en la institución educativa contemporánea con ocasión de tecnologías digitales e Internet, comprender las transformaciones actuales en contextos más amplios y describir desafíos presentes y futuros, es nuestro propósito.

\section{Usted, ¿dónde se cree que está?"}

Para comenzar, un primer punto consiste en figurar y caracterizar la institución educativa en la actualidad. Para ello se recuperan algunas de las ideas planteadas por Dubet, Francois en "El declive de la institución. Profesiones, sujetos e individuos ante la reforma del Estado" (2006). A nuestro entender, el declive del programa institucional ${ }^{4}$ cobra mayor visibilidad en la impronta, en el resultado, que la vida institucional juega, ¿jugará? en el orden social y en la eficacia al transmitir un tipo particular de conocimiento. No así, o no tan así, en el orden de lo vivenciado, experimentado por ese sujeto que la transita y, en el mismo movimiento, construye. De otra manera, la “entrada” a la vida institucional sagrada por parte de los sujetos implica que estos continúen allí, entre otra multiplicidad de espacios, su proceso de subjetivación. Por supuesto, dicho tránsito difiere al experimentado allí en épocas pasadas. Sin embargo, eso no implica, o no

\footnotetext{
4 “El programa institucional es una composición simbólica y práctica al que se pliegan aquellos que están encargados de llevarlo a cabo" (Dubet, 2007:45).
} 
conlleva a suponer necesariamente que este transitar no se objetive -"vuelva cuerpo"- en las subjetividades que por allí circulan. Más aún, y refiriéndonos específicamente al mundo escolar, si bien la institución educativa no goza ni de la legitimidad, ni de la eficacia que alcanzó en la modernidad, continúa siendo uno, no el único, de los espacios en el que la mayoría de las crías sapiens (Corea, Cristina y Lewkowicz, Ignacio; 2003) continúan instituyéndose.

En cierto sentido, entonces, elucidar acerca de la institución ${ }^{5}$ en los términos que plantea Dubet, Francois (2006) implica necesariamente referir a la acción de instituir. A ese juego pendular y en tensión constante entre lo instituido y lo instituyente que significa, por un lado, realizar un trabajo de interiorización de lo sociocultural en los soportes materiales indeterminados. Lo cual admitiría una mirada particular de la cría sapiens: la heteronomía de "ese" en formación o en estado inconcluso. Por otro, aludir a lo institucional supondría en cierto sentido que existe un conjunto de valores, principios y normas que son necesarios y valiosos de ser transmitidos.

Ahora bien, cómo se sostiene y construye el programa institucional, y todo lo que trae consigo, cuando sus andamiajes, tal como es posible identificar desde hace ya un par de décadas, comienzan a resquebrajarse, hasta por momentos estallar. Más específicamente, podemos suponer que los principios sobre los que se erigen y se (de)(re)construyen las instituciones no tengan el anclaje de antaño, sin embargo ¿ello implica una ausencia de éste? ¿O, simplemente, una invisibilización de ciertos rasgos del mismo? En términos de Sennet (2006), si este capitalismo tiene como motor lo inminente, el carecer de sentido y largo plazo, entonces, qué conservar/descartar en el vínculo intergeneracional. Uno de los juegos propios de la institución educativa entre otros.

Ensayar una posible respuesta requiere caracterizar brevemente el programa institucional de Dubet, F. (2006). A grandes rasgos, éste refiere a los valores y principios universales, organizados de forma homogénea y coherente, que posibilitan construir este

\footnotetext{
${ }^{5}$ Dada la polisemia del concepto institución vale recordar sus distintas acepciones. "Por instituciones se entiende: las formas sociales establecidas, es decir aquellas formas que remiten a lo instituido, reglado, normado; los procesos por los cuales las sociedades y los individuos se organizan, o sea que nos remite a los procesos de cambio, de transformación de lo instituido, es decir, lo instituyente; el proceso de institucionalización, que resulta del permanente interjuego y tensión entre lo instituido y lo instituyente" (Frigerio y Poggi, 1992:34).
} 
adentro y afuera de las instituciones. Así, en cierto sentido, el título de este apartado "-Y Usted, ¿dónde se cree que está?” intenta sintetizar la dicotomía sobre la que el programa institucional se asienta, se construye y edifica. Al mismo tiempo, es posible advertir que en ese mundo de lo "sagrado/escolar" el(los) conocimiento(s) válido(s) y considerados valiosos y necesarios de ser transmitidos se produce por fuera de dicho santuario. Más aún, generalmente se originan en el mundo científico y se traducen para transponerse en las instituciones educativas. Así, entonces, podríamos pensar ¿qué conocimiento(s) para ${ }^{6}$ este santuario? Si bien estos saberes se presentan como los únicos posibles, desde hace aproximadamente más de dos décadas esta visión comienza a ser repensada. De hecho, algo de ello podría estar materializándose tanto en las teorías de la enseñanza, como en las diversas transformaciones curriculares que se han suscitado, por nombrar algunos ejemplos.

Si a las características del programa institucional presentadas, le incorporamos tecnologías digitales e Internet, tal como ocurre en la mayoría de las escuelas de nuestra región, América Latina y el Caribe, a partir de los planes "una computadora, un alumno" es viable suponer posibles dislocaciones en algunas de las bases sobre las que se sustenta dicha vida institucional. Específicamente, la posibilidad de "romper" con el tiempo y espacio sincrónico escolar, el eventual ingreso de otros soportes como portadores legítimos de saber y distinto a los docentes, por ejemplo, tutoriales, netbooks, entre otros, y junto con estos, una diversidad de valores, podrían tensionar al programa institucional.

Finalmente, en este apartado, hemos reflexionado acerca de algunas de las características de dicho programa, especialmente la construcción de la institución educativa, y sus distintos actores, como espacio sagrado en el que la demarcación entre el adentro y afuera es tajante. Luego, insinuamos muy tímidamente cómo la concepción de conocimiento que allí prevalece se asienta sobre dicha separación. A precisar algo de dicha relación, nos dedicamos en las líneas que siguen.

\footnotetext{
${ }^{6}$ La preposición para intenta señalar la diferencia crucial en pensar a la institución educativa, y con ella a cada una de sus actores, como productora de algún tipo de conocimiento o, simplemente, concebirla como reproductora del mismo.
} 


\section{-¿El juego educativo por antonomasia: apropiarse del conocimiento producido por otros y, en el devenir, perder lo propio?}

La escuela moderna descansa, entre otras cuestiones, en la necesidad de homogeneizar y universalizar saberes considerados socialmente válidos. Por ello, el lugar que ha tenido la reproducción del conocimiento en la conformación y base de la institución escolar es central. Quisiéramos resaltar la idea de reproducción, ya que ésta implica un determinado posicionamiento acerca de qué es considerado saber y que no; así como, quién/es está/n habilitado/s a producirlo. Con lo cual, y en cierto sentido, permite que se sostengan, incluso hasta nuestros días, ciertas prácticas y representaciones que imposibilitan $\mathrm{y} / \mathrm{u}$ obturan considerar como tal al conocimiento que se genera en las instituciones educativas.

Claro que antes de continuar profundizando la relación entre el saber sabio y el escolar, conviene presentar qué entendemos por conocimiento. Entonces, además de advertir la existencia de diferentes concepciones acerca del mismo, es necesario señalar que una cosa es el conocimiento como ente emergente de la materia/energía y otra, muy distinta, es la forma contingente en que el conocimiento se nos presenta de manera inmediata. En otros términos, desde la mirada que aquí se propone, se considera al conocimiento a partir de su materialidad (Zukerfeld, Mariano 2010). Esto implica, en primer lugar, entender a éste como flujo de recursos y, en segunda instancia, identificar y señalar que dentro de la institución escolar, como de cualquier otra, circulan diferentes niveles del mismo.

Así, la dualidad entre el saber escolar y el saber "sabio" podría residir, entre otras cosas, no solamente en las bases del programa institucional; sino también en la concepción reduccionista del conocimiento en general. Esta distinción entre el saber producido en otros ámbitos, especialmente el científico-académico, requiere y posiciona a la escuela en particular y a los sistemas educativos en general, como administradores y transmisores de éste. Dicho juego podría, entre otras cuestiones, invisibilizar los diversos conocimientos que se constituyen y construyen a la institución educativa, que si bien exceden al acto pedagógico en sí, intervienen y circundan al mismo. En algún punto, 
dicho juego permite, entre otros, por un lado, conservar a la escuela como santuario y, por otro, conduce a que todo lo producido dentro de la misma sea la mayoría de la veces “descartado", o sea no sea considerado bajo el gran significante conocimiento. En suma, la escisión entre los espacios de (re)producción del conocimiento conlleva a que en la escuela se establezcan reglas propias de funcionamiento, no solamente en términos epistémicos, sino en el funcionamiento institucional de éste y su producción (Terigi, Flavia 2007). Claro que dicha separación excede a la escuela como "productora o reproductora" de conocimiento, sino que también delinea un vínculo y posicionamiento de los diferentes actores educativos en tanto que sujetos pasivos.

Entonces, si el lectxr comparte, tal como ya ha sido mencionado, que asistimos a una etapa del modo de producción capitalista en la que la producción y circulación de conocimientos son el eje central del crecimiento y desarrollo, vale volver a interrogarse acerca de: qué lugar se le dará en la escuela a ésta. Más aún, generalmente, el mundo de la educación formal no se (auto)concibe como espacio de producción de conocimientos. Así, puede que estemos transitando una etapa en la que sea la transmisión quien comience a jugar el centro de la escena escolar. En términos de Dicker, Gabriela (2004:224) "lo propio de la transmisión es que ofrece a la vez una herencia y la habilitación para transformarla". Obviamente, podría conjeturarse que ésta es una mera cuestión de intercambio de conceptos, no obstante consideramos que no lo es ya que dicho posicionamiento supone una concepción del acto educativo diferente, donde no solamente "ese" y eso dado requieran ser transformados y apropiados; sino que, al mismo tiempo, implica concebir al otro, en cualquiera de sus roles institucionales, como potencia, con la capacidad de transformación.

Por supuesto, aquí no se propone una homologación de los diferentes tipos de conocimiento que conviven en la institución educativa, sino de la necesidad de advertir que estos distintos tipos de saberes se (re)producen y circulan por canales que requieren ser diferenciados.

En resumen, sostener una mirada monolítica del conocimiento y con ella de la reproducción de éste fue una de las razones del programa institucional. Sin embargo, podríamos asistir a una etapa en la que la (re)producción de conocimientos, junto con un 
sujeto capaz de (re)producirlo, sea central en los diferentes órdenes de la vida social. Lo cual podría implicar una transformación en la concepción y producción del conocimiento.

\section{-Algunos apuntes acerca de la vida institucional, los conocimientos y las tecnologías digitales e Internet}

Tal como hemos señalado, en el capitalismo informacional los conocimientos se producen y circulan cada vez con mayor injerencia y rapidez a través de tecnologías digitales e Internet. En dicho contexto, el rol de la escuela como (re)productora, y con ella de la educación formal en su conjunto, está siendo repensado.

En este sentido, se concibe que la incorporación de este tipo de tecnologías tensiona, hackea, los ejes fundamentales del programa institucional. Se intenta figurar en términos de posibilidad, no de que efectivamente esto haya ocurrido o que esté aconteciendo, sino en que esta incorporación a la institución escolar abre la posibilidad de resituar aquello, que hasta ahora, ha sido denominado bajo el significante escuela. Como señala Barbero, Martín (2002:2)

las tecnologías digitales deslocalizan los saberes modificando tanto el estatuto cognitivo como institucional de las condiciones del saber y las figuras de la razón. Es disperso y fragmentado como el saber puede circular por fuera de los lugares sagrados que antes lo detentaban y de las figuras sociales que lo administraban.

Pero, ¿qué de particular presentan estas tecnologías? Las mismas permiten producir, copiar y distribuir conocimiento en soporte digital con un costo cercano a cero. Lo que comúnmente ha dado en llamarse la "replicabilidad del bit" (Cafassi, Emilio 1998). Asimismo, son disruptivas en términos de espacio y tiempo, ya que permiten romper con las barreras espaciales y tornan todo tiempo susceptible de ser productivo, o sea se difumina la diferenciación entre el tiempo de trabajo y el tiempo de ocio, o lo que suele denominarse una imbricación entre economía y cultura. Dichas características conviven, se ponen a dialogar, con dos rasgos particulares de Internet: por un lado, su reticularidad, 
la forma en red potencia la cantidad de conocimiento disponible y, por otro, su acentrismo, dificulta la limitación que circula en la red (Vercelli, Ariel 2006).

Ahora bien, la problematización que se intenta presentar aquí vincula, por un lado, las tecnologías digitales e Internet con una de las características del programa institucional - el santuario- $y$, por otro, busca reflexionar acerca de las posibles consecuencias de este desfasaje entre soportes analógicos y digitales. Lejos de encontrarnos en una situación de certezas, se comparten aquí algunas de las inquietudes que nos atraviesan.

La primera de ellas reside en torno a la escisión entre el adentro y afuera del santuario escolar. Así, la incorporación de las tecnologías digitales e Internet, en cualquiera de sus variantes, como, netbook, celular, entre otras, podría acrecentar la posibilidad de intromisión del mundo profano en el sagrado. Más aún, si consideramos que con dichos dispositivos se puede navegar, bajar e intercambiar una diversidad de conocimientos que no necesariamente se corresponden con aquellos legitimados por la escuela, ni con los producidos por los espacios autorizados para ello, podrían generarse con dicha inclusión una diversidad de tensiones. Algunas posibles se suscitan entre los actores de la institución educativa, por ejemplo, docentes y estudiantes que buscan información acerca de lo trabajado y repreguntan en función de dicho saber; entre los conocimientos considerados válidos, porte al mencionar algunos.

La segunda cuestión descansa en la posibilidad de compartir aquello que se produce en la escuela, su visibilidad y las reestructuraciones que esta posibilidad trae. Quiero decir, la escuela moderna en algún sentido asentó sus prácticas en que lo allí enunciado quedaba allí, no se divulgaba, a lo sumo, y en el mejor de los casos, algún docente o directivo transmitiría su experiencia. Sin embargo, con la llegada de las tecnologías digitales e Internet se abre un abanico de canales de comunicación, difusión e intercambio. Por supuesto, habrá que rastrear, entre otras diversas cuestiones, qué consecuencias trae a la vida institucional y para cada uno de los actores educativos este tipo de intercambios. 
En síntesis, puede que la cruzada que se libra entre el programa institucional - el santuario- y las tecnologías digitales e Internet, entre otras muchas, radique en intentar sostener y conservar ese lugar de reproducción de los conocimientos sobre el que se construyó y edificó la institución educativa, para empezar a ubicarse, u oficiar de productora e intermediaria. Ese cambio de rol, en cierta manera, atenta contra una de las bases del programa institucional, así como el lugar que sus actores, especialmente docentes y estudiantes, han jugado a lo largo de siglos. Sin embargo, estos arribos a la institución educativa y con ella a la vida de cada uno de los sujetos que por allí se (de)(re)construyen podrían ser los que posibiliten (re)edificar espacios cada vez más potentes.

\section{- A modo de cierre}

A lo largo de estas páginas hemos insinuado que el programa institucional (Dubet, Francois2006) experimenta, hace ya aproximadamente tres décadas, dislocaciones y tensiones de las más diversas. Claro que aquí solamente hemos intentado dar cuenta de algunas de ellas. Las mismas son percibidas, vivenciadas, las más de las veces, como una suerte de orfandad de los sujetos. Si bien no es el motivo de este ensayo detenernos en dicha sensación, creemos que es válido señalar, o no dejar de advertir, que el juego pendular entre lo instituido y lo instituyente, o de ese "ir y venir" de la vida institucional, abre la posibilidad -latente, claro está- que tienen los sujetos con su capacidad de agencia de tejer redes o, simplemente, ampliar las ya disponibles.

Hecha la salvedad, y en función de lo compartido en este ensayo, una de las cuestiones que nos interesa recuperar es acerca del "trabajo sobre los otros". Entonces, puede que a partir de las características atribuidas a esta etapa del capitalismo, junto con las diversas tensiones que atraviesa el programa institucional, dicho trabajo comience a ser entendido como un "trabajo con los otros". Este cambio preposicional parece anecdótico, sin embargo, creemos que no lo es, ya que implica concebir la heterogeneidad de los sujetos, junto con sus diversos conocimientos. Algo de esto se 
identifica en el trabajo colaborativo y en todo aquello que se engloba como la web 2.0. Puede entonces que la entrada de (re) productores y consumidores digitales convoquen a nuevos interrogantes, algunos posibles, ¿qué disciplinas están disputando la vanguardia? ¿Qué conceptualizaciones? ¿Quiénes oficiarán de responsables en este nuevo juego?

Finalmente, desde la perspectiva propuesta aquí, señalar algunas posibles dislocaciones del programa institucional conlleve a figurar su finitud en algún devenir impostergable. Sin embargo, por estos días, lejos de resolverse y establecerse posibles salidas a la vida ofertada y construida por $y$ en instituciones con fines ya predeterminados, presenciamos la estabilización de una vida institucional con más desajustes que acuerdos. En este sentido, el horizonte que inunda nuestro tránsito cotidiano está íntimamente relacionado con esta multiplicidad de horizontes en los que la única de las certezas descansa en el devenir de la incertidumbre. Puede entonces que ila estructura estable de lo institucional radique en ella, en la capacidad de esta estructura de moldearse continuamente?

\section{Referencias}

CAFASSI, Emilio. Bits, moléculas y mercancías, en FINQUELIEVICH, S. y SCHIAVO, E. (Comp.) La ciudad y sus TICS: tecnologías de información y Comunicación. Bernal, Universidad Nacional de Quilmes, 1998.

BLONDEAU, Oliver. Génesis y subversión del capitalismo informacional, en RODRÍGUEZ, Emanuel y SÁNCHEZ, Raúl (Compiladores) Capitalismo cognitivo, propiedad intelectual, y creación colectiva, Madrid, Traficantes de sueños, 1999.

BARBERO, Jesús Martín. La educación desde la comunicación, Bs. As., Norma, 2002.

BUCKINGHAM, David. Más allá de la tecnología. Aprendizaje infantil en la era de la cultura digital. Buenos Aires, Manantial, 2007.

CASTELLS, Manuel. La era de la información, tomos I, Il y III, Siglo XXI, México D.F., 1997.

COREA, Cristina y LEWKOWICZ, Ignacio. ¿Se acabó la infancia? Ensayo sobre la destitución de la niñez. Buenos Aires, Editorial Lumen/Hvmanitas, 1999. 
DIKER, Gabriela. Y el debate continúa. ¿Por qué hablar de transmisión?, En FRIGERIO, Graciela y DIKER, Gabriela. (Comps.) La transmisión en las sociedades, las instituciones y los sujetos. Un concepto de la educación en acción, Buenos Aires, Novedades Educativas, 2004.

DUBET, François. El declive de la institución: Profesiones, sujetos e individuos ante la reforma del Estado, Barcelona, Gedisa, 2006.

DUBET, François. Conflictos de normas y ocaso de la institución, en Estudios sociológicos, enero-abril/vol. XXII, nº. 001, El Colegio de México, DF. Pp. 3-24, 2004.

DUSSEL, Inés. Aprender y enseñar en la cultura digital, Documento Básico - Fundación Santillana, 2011.

DUSSEL, Inés. y QUEVEDO, Luis Alberto. Educación y nuevas tecnologías: Los desafíos pedagógicos ante el mundo digital, VI Foro Latinoamericano de Educación, Fundación Santillana, 2010.

FRIGERIO, Graciela. y POGGI, M. Cara y Ceca: F (1999).

SENNETT, Richard. La cultura del nuevo capitalismo, Barcelona, Anagrama, 2006.

TERIGI, Flavia. La enseñanza como problema político, en FRIGERIO, Graciela y DIKER, Gabriela. (Comps.) La transmisión en las sociedades, las instituciones y los sujetos. Un concepto de la educación en acción, Buenos Aires, Novedades Educativas2004.

TERIGI, Flavia. Exploración de una idea. En torno a los saberes sobre lo escolar, en BAQUERO, Ricardo, DIKER, Gabriela y FRIGERIO, Graciela. Las formas de lo escolar, del estante editorial, Buenos Aires, 2007.

VERCELLI, Amilcar. Aprender la libertad, 2006.

ZUKERFELD, Mariano. Capitalismo y conocimiento, Tesis Doctoral - FLACSO, Bs. As., 2010. 